\title{
Die Krise Frunda: Das Dilemma der ungarischen Rumänen
}

\author{
Heiko Fürst*
}

\begin{abstract}
Taking as its starting point the recent Romanian controversy over majority-minority and state-nation relations, this article explores the political representation of ethnic Hungarians in Romania. It critically reviews the history of the DAHR, the main political organisation of Hungarians in Romania, from its founding to the present day, discussing the DAHR's constitutional principle of including various platforms under a single roof, analysing the major actors and their struggles for influence within the alliance. Reflecting the fact that the role played by ethnic Hungarians in Romanian political life can be decisive, the conclusion takes the results of the analysis to make more general remarks on political co-operation and future inter-ethnic relations.
\end{abstract}

Keywords: Rumänien, Minderheiten, Parteiensystem, Ungarn, interethnischer Konflikt

$\mathrm{D}$ ie interethnischen Befindlichkeiten in Rumänien verschärften seit dem letzten Regierungswechsel erneut die politische Situation in Rumänien. Mitte Februar 2006 rief der Vorsitzende des Verbands der ungarischen Minderheit in Rumänien (RMDSZ) Staatspräsidenten Băsescu dazu auf, mäßigend in die Debatte einzugreifen. Der hingegen hatte bereits im Monat zuvor nicht vermocht, beruhigend auf die erhitzten Gemüter der Demokratischen Partei (PD) und des Ungarnverbands einzuwirken, die sich gegenseitig mit der Aufkündigung der gemeinsamen Regierungskoalition drohten. Streitpunkt war wieder einmal das nationale Selbstverständnis Rumäniens und die Frage nach dem Status der Minderheiten, die Rumänien seit 16 Jahren in regelmäßigen Abständen in signifikante Regierungskrisen treibt.

Im Januar 2006 hatte eine Debatte im Europarat die Animositäten zum Höhepunkt getrieben. Verabschiedet worden war die Resolution 1735, die sich mit dem Konzept der »Nation « befasste und Empfehlungen für die Mitgliedstaaten formulierte. ${ }^{1}$ Berichterstatter war György Frunda, ethnischer Magyare und Leiter der rumänischen Delegation in der Parlamentarischen Versammlung. Die verabschiedete Resolution erklärte die Definition ethnisch einheitlicher Staaten für unzeitgemäß und forderte die Länder des Europarats dazu auf, ihre Verfassungen mit europäischen Standards in Einklang zu bringen und den Staat nicht entlang ethnisch exklusiver Kriterien zu konstituieren.

Obwohl die Resolution nicht verbindlich ist und Frunda in der Debatte vorsichtiger formulierte als sein nichtungarischer Delegationsvize, löste die Verabschiedung in Rumänien eine Protestwelle aus. Emil Boc, Vorsitzender der Demokratischen Partei, erklärte den Vorgang zu einem Angriff auf die Verfassung. Frunda müsse sofort aus der Delegation abberufen werden. Sollte dies nicht geschehen, müsse der Ungarnverband die Regierungskoalition verlassen. Das Leitungsgremium der Allianz der beiden größten Koalitionsparteien beschloss,

\footnotetext{
* Heiko Fürst M.A., Institut für Friedensforschung und Sicherheitspolitik an der Universität Hamburg (IFSH) und Institut für Politikwissenschaft der Universität Leipzig. Dieser Aufsatz wurde referiert.

1 Parliamentary Assembly of the Council of Europe: The concept of »nation «, Recommendation 1735 (2006). http://assembly.coe.int/Main.asp?link=/ Documents/AdoptedText/ta06/EREC1735.htm
}

György Frunda seines Amtes als Delegationsleiter zu entheben. ${ }^{2}$ Der stellvertretende Vorsitzende der oppositionellen Sozialdemokratischen Partei warf dem RMDSZ vor, Rumänien beständig bei europäischen Gremien anzuschwärzen. ${ }^{3}$ Nachdem Frunda darüber hinaus beschuldigt wurde, in einem Interview geäußert zu haben, dass er Rumänien nicht repräsentiere, forderten Vertreter der Konservativen Partei, ein Strafverfahren gegen ihn einzuleiten. ${ }^{4}$

Der Parteichef des RMDSZ, Béla Markó, bestärkte in der Folge die Resolution und Frundas Bericht, indem er konstatierte, niemand könne heute davon sprechen, dass Rumänien der Staat einer einzigen Nation sei. Im Übrigen verfüge die Regierung ohne den Ungarnverband nicht über eine parlamentarische Mehrheit, und seine Partei müsse sich keine Direktiven von anderen gefallen lassen. ${ }^{5}$ Emil Boc bezichtigte er eines ungarnfeindlichen Diskurses und verglich ihn mit dem ehemaligen nationalistischen Bürgermeister von Cluj, dessen Amt er 2004 übernommen hatte. ${ }^{6}$ Der RMDSZ-Abgeordnete Kelemen Hunor rief zu Einheit und Standhaftigkeit auf, damit garantiert sei, dass die Ungarn nicht als »Verlierer« aus dem Konflikt hervorgingen. ${ }^{7}$ Nachdem der Ungarnverband seine Position durchsetzen konnte, titelte die ungarische Minderheitenpresse kampfeslustig: Ungarnverband - Demokratische Partei 1:0. ${ }^{8}$

Die Kontroverse um György Frunda wirft indessen ein bezeichnendes Licht nicht nur auf die Konfliktlinien zwischen den Volksgruppen, sondern insbesondere auch auf die Verwerfungen innerhalb der ungarischen Gemeinschaft selbst. Durchhalteparolen und populistische Vergleiche wie sie der

2 Frunda-Resolution in der PVER erregt in Bukarest die Gemüter, in: Allgemeine Deutsche Zeitung, 8.2.2006.

3 Oana Dan: Politician lashes Hungarian Alliance senator for "non-constitutional attitude«, in: Bucharest Daily News, 6.2.2006.

4 Andreea Pocotila, UDMR head: "We do not accept lessons in loyalty«, in: Bucharest Daily News, 8.2.2006.

5 Az RMDSZ nélkül nincs többségi koalíció [Ohne den RMDSZ gibt es keine Koalitionsmehrheit], in: Magyar Hírlap, 7.2.2006.

6 Ana Serban, György Frunda: PD would like to hold early elections after the country report in May, in: Nine o' Clock, 14.2.2006.

7 Nincs koalíciós egyezség sem Frundáról, sem a kisebbségi törvénytervezetrôl [Keine gemeinsame Position der Koalition zu Frunda und dem Entwurf eines Minderheitengesetzes], in: Szabadság, 8.2.2006.

8 Román sajtóvisszhang: RMDSZ-DP 1-0 [Rumänisches Presseecho: RMDSZPD 1:0], in: Szabadság, 9.2.2006. 
den moderaten Kräften zuzurechnende Parteichef Markó verlautbaren ließ, sind ein Indiz des Dilemmas, in dem sich der Ungarnverband befindet. Dieses liegt in seinem offenen Organisationsprinzip begründet, das einerseits als Vorteil bei der Regelung interethnischer Konflikte angesehen wurde, andererseits aber auch zeit seines Bestehens zu ausgeprägten innerparteilichen Konflikten führte, was seine Stabilität gefährdete und bisweilen zur Einnahme kompromissloser Positionen des Verbands in der Regierungsverantwortung beitrug.

Im Folgenden werden diese Charakteristika sowie zentrale Streitfragen innerhalb des RMDSZ herausgearbeitet. Angesichts der Entwicklungen der letzten Jahre liegt ein Fokus der Analyse auf dem Grad an Integrationskraft, die dem RMDSZ heute noch zugesprochen werden kann. Diese Fähigkeit kann als entscheidender Indikator der Konfliktträchtigkeit der künftigen interethnischen Beziehungen in Rumänien gelten.

\section{Ein Dach für alle - der Kunstgriff der unga- rischen Rumänen}

Mit der Gründung des RMDSZ am 25. Dezember 1989 strebten die ungarischen Rumänen eine einheitliche Interessenvertretung an, die ihre aus der Zeit des Nationalkommunismus herrührende Diskriminierung beenden sollte. Formal wurde der RMDSZ nicht als Partei, sondern als Dachorganisation der verschiedenen Vereinigungen der magyarischen Rumänen geführt. Er basiert daher auf dem Prinzip des internen Pluralismus. Innerhalb des Verbandes existiert ein inhaltlich breites Spektrum so genannter »Plattformen «, die nach ihren jeweils eigenen Organisationsregeln funktionieren. ${ }^{9}$ Neben der Verbandszentrale in Bukarest gibt es ein Exekutivbüro in Cluj und starke Regionalorganisationen. Den Verband strukturieren fünf Organe: der Kongress (oberstes Entscheidungsorgan), der Repräsentantenrat (oberstes Entscheidungsorgan zwischen den Kongressen), der Schlichtungsrat (vermittelndes Gremium), der Operativrat (Entscheidungsträger in speziellen politischen Fällen) und das Präsidium (Geschäftsführung). Dem etwa eine halbe Million Mitglieder umfassenden RMDSZ gelang es durch die lose Organisationsform, die Magyaren Rumäniens quasi geschlossen hinter sich zu bringen, weshalb Anneli Ute Gabanyi von einer "mitgliederstarken Volks-,Partei' « sprach. ${ }^{10}$ Da Minderheitenorganisationen bei Wahlen zum Parlament Parteien gleichgestellt werden, konnte die Organisation bislang bei allen Parlamentswahlen erfolgreich antreten und bildet eine eigene Fraktion im rumänischen Abgeordnetenhaus. Die Differenzen innerhalb des Bundes sind aber mannigfaltig. Die selbst als Mitglieder geführten Politologen Imre Borbély und Zsolt Attila Borbély kristallisieren vier programmatische Hauptströmungen heraus: ${ }^{11}$

9 RMDSZ: Statute of the Democratic Alliance of Hungarians in Romania. Adopted by the $4^{\text {th }}$ Congress of the DAHR. Cluj (Kolozsvar), the $26-28^{\text {th }}$ of May 1995. III, C, 22, in: Democratic Alliance of Hungarians in Romania: Documents 4. Cluj 1995. S. 29-49.

10 Anneli Ute Gabanyi: Politische Parteien in Rumänien nach der Wende, in: Südosteuropa, 1-2/1995. S. 1-50 (44).

11 Imre Borbély, Zsolt Attila Borbély: RMDSZ: érték és hatalom, 1989-1998 [RMDSZ: Werte und Macht, 1989-1998], in: Magyar Kisebbség, 2/1998.
Tabelle 1: Programmatische Hauptströmungen des RMDSZ

\begin{tabular}{ccc} 
& kommunitarisch & individualistisch \\
\hline emanzipativ & 1) Autonomisierung & 3) Weltbürgertypus, \\
der ungarischen & Kemeinschaft \\
(altruistische und & Existenzgrundlage \\
moralische & \\
Begründungen) & \\
kollaborativ & 2) $\begin{array}{c}\text { ungarische Ge- } \\
\text { meinschaft als } \\
\text { abhängig von der } \\
\text { Gesellschaft }\end{array}$ & $\begin{array}{l}\text { 4) Untertanentypus, } \\
\text { keine Verantwortung } \\
\text { für die ungarische }\end{array}$ \\
& Gemeinschaft
\end{tabular}

Quelle: eigene Darstellung, nach den Angaben in: Borbély/ Borbély (Anm. 10)

Die Hauptkonfliktlinie verlief seit 1989 zwischen den beiden kommunitarischen Strömungen. Diese gruppierten sich um die beiden Zentralfiguren László Tőkés, den nach dem Systemwechsel zum Bischof ernannten Sprecher der Radikalen (Strömung 1), und den seit 1993 den Verband führenden Béla Markó als Sprecher der Moderaten (Strömung 2). Programmatisch strebte der RMDSZ zunächst die Durchsetzung umfassender Minderheitenrechte an. Durch administrative Dezentralisierung sollten vor allem zwei Ziele erreicht werden: ungarischsprachige Bildung »from the kindergarten to the native language university «12 sowie weitreichende Selbstbestimmung in Form verschiedener Arten von Autonomie. Beide Gruppierungen teilten diese Forderungen, schieden sich aber in der Frage ihrer Umsetzung.

\section{Ethnische Bipolarität - die frühen Konflikte}

Während der ersten Monate nach der Machtübernahme pflegten der Ungarnverband und die regierende Front der Nationalen Rettung (FSN) durchweg kooperative Beziehungen. Die neue Regierung, an der auch ethnische Ungarn beteiligt waren, sagte den Minderheiten die Garantie von Individualund Kollektivrechten zu. Ministerpräsident Petre Roman verkündete die Wiedereröffnung einer eigenständigen staatlichen ungarischen Universität, und in Cluj wurde das Rathaus dreisprachig beschildert. Bereits im Frühjahr 1990 traten allerdings erste Konflikte zutage. Als der Ungarnverband seine Forderungen nach Stärkung der Minderheitenrechte durchzusetzen versuchte, fand die Kooperationsbereitschaft der Regierung ein schnelles Ende. Im Zuge des Wahlkampfs griff die FSN auf die fremdenfeindliche und nationalistische Rhetorik des Ceauşescu-Regimes zurück. Nach den Wahlen im Mai trat der RMDSZ dem Oppositionsbündnis »Demokratische Konvention« bei, dem er bis 1995 angehörte.

Eine Minderheiten missachtende Politik der neuen Regierung, die in den Jahren 1994 und 1995 in der Beteiligung nationalistischer Parteien gipfelte, stärkte den radikalen Flügel innerhalb des RMDSZ bis zur Mitte der 1990er Jahre: Noch auf

12 RMDSZ: Fundamental Principles of the Programme of the Democratic Alliance of Hungarians in Romania. Adopted by the $4^{\text {th }}$ Congress of the DAHR. Cluj (Kolozsvár), the 26-28 ${ }^{\text {th }}$ of May 1995, in: RMDSZ (Anm. 9). S. 10. 
dem zweiten Verbandskongress im Mai 1991 erlitt das radikale Lager mit einer abgewiesenen Beschlussvorlage zur ethnischen Autonomie eine Niederlage. Auch ihre Forderung nach einem Referendum über Territorialautonomie für das Széklerland im Oktober 1991 fand keine breite Zustimmung. Nach den Wahlen im September 1992 jedoch verbuchten sie Erfolge. Die im Oktober verabschiedete »Deklaration von Cluj « forderte verschiedene Formen kollektiver Autonomie für die ungarische Gemeinschaft als eigenständigem politischem Subjekt. Auf dem Verbandskongress im Januar 1993 in Braşov konnten die radikalen Vertreter innerhalb des RMDSZ ihre Position stärken und ihre Forderungen forcieren. Auf ihre Initiative hin entstand ein Entwurf zu einem Minderheitengesetz. Zwar wurde in Braşov dennoch der gemäßigte Béla Markó zum Verbandspräsidenten gewählt, dies beruhte allerdings auf einem »Kompromiss«, der den gemäßigten Politikern ein radikales Programm zur Umsetzung vorgab. ${ }^{13}$

Auch die Auseinandersetzung mit dem politischen Gegner verschärfte sich bis zur Mitte des Jahrzehnts. Mit dem Gesetz zur lokalen Administration wurden im November 1991 die Rechte zur Nutzung der Muttersprache in der örtlichen Verwaltung beschränkt. Im Folgemonat verabschiedete das Parlament gegen die Stimmen der ungarischen Vertreter und einiger rumänischer Abgeordneter (inkl. des Justizministers) eine neue Verfassung, die Rumänien als unteilbaren Nationalstaat definierte. ${ }^{14}$ Die kontinuierlichen Proteste des Ungarnverbands führten zu einer Polarisierung der Gesellschaft zwischen Mehrheit und Minderheit. Die vor allem vom radikalen Flügel lautstark vorgetragene Forderung nach kollektiven und territorialen Autonomierechten brachte dem RMDSZ die Feindschaft der rumänischen Parteien und der populistischen Presse ein. In einer Studie des Meinungsforschungsinstituts CIS (Centrul Independent de Sondaje) vom August 1992 zählten über 30\% der Befragten den Ungarnverband zur extremen Rechten. ${ }^{15}$ Drei Jahre später stuften ihn $80 \%$ der Rumänen als anti-rumänisch ein. ${ }^{16} 1995$ eskalierte die Debatte, so dass der Ungarnverband selbst das Oppositionsbündnis verlassen musste. Nachdem der Vorsitzende der Demokratischen Konvention, Emil Constantinescu, den Magyaren ein Ultimatum gestellt hatte, die rumänische Verfassung und Rumänien als »einheitlichen und unteilbaren Staat « anzuerkennen ${ }^{17}$, verließ der RMDSZ am 26. Februar 1995 die Allianz.

Mit Blick auf die Forderungen des RMDSZ spricht der FSN-Aktivist Vladimir Pasti von einem Prozess, bei dem Konflikte be-

13 István Horváth: Facilitating Conflict Transformation: Implementation of the Recommendations of the OSCE High Commissioner on National Minorities to Romania, 1993-2001. Hamburg 2002. S. 33.

14 So der dem radikalen Flügel zuzurechnende Géza Szőcs im Januar 2000 (Géza Szőcs; Mária Gál: 10 éves az RMDSZ. Interjú Szőcs Gézával [10 Jahre RMDSZ. Interview mit Géza Szőcs], in: Szabadság, 14.1.2000. S. 3, 6).

15 Zur Diskussion der Verfassung siehe Renate Weber: The Protection of National Minorities in Romania: A Matter of political Will and Wisdom, in: Jerzy Kranz (Hg.): Law and Practice of Central European Countries in the Field of National Minorities Protection after 1989. Warszawa 1998. S. 199-268 (201ff); István Horváth, Alexandra Scacco: From the Unitary to the Pluralistic: Fine-Tuning Minority Policy in Romania. http://lgi.osi.hu/publications/ books/Diversity_in_Action/2_5.PDF. S. 253ff; Janusz Bugajski: Ethnic Politics in Eastern Europe. A Guide to Nationality Policies, Organizations and Politics. New York, London 1994. S. 207f.

16 Alina Mungiu: Die Rumänen nach »89. Sozio-politische Studie. Reşiţa 1996. S. 120 .

17 Günter Klein: Rumäniens Minderheitenpolitik im Kontext internationaler Beziehungen und Empfehlungen des Europarates, in: Südosteuropa 11-12/1996. S. 815-839 (827). wusst intensiviert wurden, um die »Selbst-Enklavisierung « der ungarischen Gemeinschaft zu erreichen und entscheidende administrative Positionen selbst besetzen zu können. ${ }^{18}$ Pastis Analyse gilt sicherlich für Vertreter der radikalen Gruppe im RMDSZ, die im Herbst 1992 im Verband an Terrain gewann und ihre Macht bis 1995 ausbauen konnte. ${ }^{19}$ Dennoch simplifiziert die Aussage in zwei Punkten: Erstens missachtet sie die Komplexität der Verbandsstruktur, in der Protagonisten wechselseitig inkonsistenter Positionen um Einfluss ringen. Zweitens zeugt gerade die Tatsache der Regierungsbeteiligung und die während dieser Zeit auch in nicht minderheitsbezogenen Politikfeldern geleistete Arbeit vom Integrations- und Gestaltungswillen des Verbands. Auch rumänische Analysten wie Bogdan Murgescu von der Universität Bukarest bescheinigen dem RMDSZ, bislang eine hervorragende Regierungsarbeit geleistet zu haben. ${ }^{20}$

\section{Konflikte über die Bildung}

Das Bildungswesen war seit 1990 ein ebenso zentrales wie problembehaftetes Anliegen des RMDSZ. Da die Regierung im Mai 1990 das bislang geltende Bildungsgesetz suspendiert hatte, blieb das Bildungswesen bis 1995 ohne Regelung. Die Situation der Minderheiten war hierbei besonders kritisch, da die Verfassung 1991 festsetzte, dass »education of all grades shall be in Romanian « und die Details in ein separates Gesetz auslagerte. ${ }^{21}$ Dem ersten Entwurf im Jahr 1992 folgten über 2000 Änderungsanträge. ${ }^{22}$ Der inzwischen klar von der radikalen Gruppe gesteuerte Ungarnverband initiierte 1994 einen eigenen Antrag, den das Parlament allerdings zu diskutieren ablehnte. Das im Sommer 1995 in Kraft getretene neue Gesetz, das die minderheitensprachliche Schulausbildung beschränkte und die Gründung eigenständiger Universitäten verbot, löste beim RMDSZ Entrüstung aus. Der Verband legte Verfassungsbeschwerde ein und protestierte vor verschiedenen internationalen Gremien. Obwohl europäische Organisationen einstimmig Nachbesserungen für dringend erforderlich hielten, versuchten sie auch beschwichtigend auf die aufgeheizte Situation einzuwirken. So erklärte der OSZE-Hochkommissar für Nationale Minderheiten, das Gesetz sei mit internationalen Normen prinzipiell vereinbar, entscheidend sei nun aber die Art seiner Umsetzung. ${ }^{23}$ Die im Ungarnverband dominierenden radikalen Vertreter ließen sich hierdurch jedoch nicht besänftigen.

Mit dem vierten Verbandskongress im Jahr 1995 verlor der Konflikt zunächst an Schärfe, da es den moderaten Kräften gelang, Einfluss im Verband zurückzugewinnen. ${ }^{24}$ Imre und

18 Vgl. Wolfgang Zellner, Pál Dunay: Ungarns Außenpolitik 1990-1997. Zwischen Westintegration, Nachbarschafts- und Minderheitenpolitik. BadenBaden 1997. S. 259f

19 Vladimir Pasti: The Challenges of Transition. Romania in Transition. New York 1997. S. 199ff.

20 Imre Borbély, Zsolt Attila Borbély (Anm. 11).

21 Bogdan Murgescu: Unveröffentlichter Vortrag zur politischen Landschaft Rumäniens. Bukarest, 21.9.2005.

22 Constitution of Romania. Art. 32 (2) und 32 (3).

23 Cesar Birzea: Educational Reform and Power Struggles in Romania, in: European Journal of Education 1/1996. S. 97-107 (102).

24 Max van der Stoel: Statement by the OSCE High Commissioner on National Minorities, on the occasion of his mission to Romania on 28-31 August 1995. http://www.osce.org/press_rel/1995/09/818-hcnm.html 
Zsolt Attila Borbély kritisierten dies als einen »Putschversuch « der Personen um Béla Markó und identifizierten zwei Tendenzen: (1) die Zunahme der Kluft zwischen politischen Programmaussagen und tatsächlichem Handeln sowie (2) eine »interne Oligarchisierung «, d.h. die Stärkung zentraler Institutionen auf Kosten pluralistischer Gremien der Basis wie dem als Verbandsparlament fungierenden Repräsentantenrat. ${ }^{25}$ Der einstige Gründer von Radio Free Europe in Budapest und spätere RMDSZ-Abgeordnete, Géza Szőcs, teilte diese Diagnose, charakterisierte sie allerdings als generelles Problem des Ungarnverbands, bei dem es wenigen Personen gelinge, die Richtung der gesamten Organisation zu bestimmen und »über das Schicksal von 2 Millionen Menschen zu entscheiden ${ }^{26}{ }^{26}$

Als paradigmatisch für die Kursänderung kann die Regierungsbeteiligung nach dem Wahlsieg der Opposition im November 1996 angesehen werden. Während moderate Akteure in der Folge die Errungenschaften in der Koalition hervorhoben (Korrekturen am Bildungsgesetz, am Gesetz über die lokale Verwaltung, Sensibilisierung für die Minderheitenfrage), protestierten die Radikalen gegen vermeintlich erreichte Ergebnisse und strebten nach Durchsetzung insbesondere der Autonomieforderungen. ${ }^{27}$

Obwohl die für die Minderheitenbildung restriktivsten Passagen des Bildungsgesetzes 1997 modifiziert worden waren, lösten interne Differenzen in der Regierungskoalition, gepaart mit erneut zunehmenden Spannungen innerhalb des Ungarnverbands eine wiederholte Krise über die Bildungspolitik aus. So stimmte der zuständige Senatsausschuss im November 1997 gegen die in Form einer Gesetzesverordnung beschlossenen Änderungen am Bildungsgesetz, woraufhin der Ungarnverband einen »Regierungsstreik « ausrief. Im Jahr darauf votierten Vertreter der Koalitionsparteien im Senat mit der Opposition mehrheitlich gegen die veränderten Passagen des Bildungsgesetzes. Dies führte die Regierung erneut in eine Bestandskrise, da der Repräsentantenrat des RMDSZ unter dem Druck der radikalen Kräfte den Regierungsaustritt beschloss. Dieser konnte zwei Stunden vor Inkrafttreten durch die Einigung auf die Gründung einer multikulturellen deutsch-ungarischen Universität gerade noch verhindert werden. Die Universität wurde nie gegründet, doch hielt der Kompromiss bis zum Ende der Legislaturperiode.

\section{Polarisierung und Zerfall}

Nach den Wahlen im Herbst 2000 trat der RMDSZ nicht mehr in die Regierung ein. Er blieb seiner moderaten Linie aber treu, indem er eine Unterstützungsvereinbarung für eine Minderheitenregierung der Sozialdemokratischen Partei (PSD) unterzeichnete. Die Vereinbarung war an die Umsetzung bestimmter Ziele gebunden. ${ }^{28}$ In dieser Legislaturperiode konnten einige

25 Dieser Wechsel entsprach auch der Stimmung in der ungarischen Bevölkerung Rumäniens (Tom Gallagher: Controversy in Cluj, in: Transition 15/1995. S. 58-6 (59)).

26 Imre Borbély, Zsolt Attila Borbély (Anm. 11).

27 Géza Sző́cs, Mária Gál (Anm. 14).

28 Vgl. István Székely: A belsố választások és az RMDSZ hatalmi szerkezetének összefüggései [Die innerverbandlichen Wahlen und die Zusammenhänge der Machtorganisation des RMDSZ], in: Magyar Kisebbség 1/2000.
Erfolge verbucht werden: Mehrere während des Kommunismus verstaatlichte Immobilien wurden rückübertragen, die Rechte der Minderheiten in der kommunalen Verwaltung wurden verbessert, die Babeş-Bolyai Universität in Cluj multikulturell umgestaltet und die von der Sapientia-Stiftung initiierte ungarische Privatuniversität erhielt ihre Akkreditierung.

Die radikale Plattform innerhalb des RMDSZ beklagte zunehmend ihren Einflussverlust und drohte mit Abspaltung. ${ }^{29}$ Dennoch gelang es der Führung zunächst, die Mitglieder entlang des Konzepts Ethnos zu organisieren und den Zerfall zu verhindern. ${ }^{30}$ Verbandspräsident Markó stellte die »Repräsentation der ungarischen Gemeinschaft in Rumänien und die Verteidigung ihrer Interessen $\aleph^{31}$ in den Vordergrund und beschwor den harten Kern einer »ethnischen Vereinigung « ${ }^{32}$, bei der galt: Wir haben noch immer viel zu verlieren. ${ }^{33}$

Mit zunehmender Dauer der Regierungsverantwortung verschärften sich die internen Spannungen zwischen der Parteiführung und der radikalen Plattform allerdings zusehends. Nachdem die führenden Köpfe der Radikalen, László Tőkés und Tibor Toró T., jahrelang gegen die als zu kompromissbereit angeprangerte Verbandspolitik opponiert hatten, folgten im Frühjahr 2003 erste Schritte der Spaltung. Anlässlich des ungarischen Nationalfeiertags am 15. März berief ein Bürgerforum einen Initiativrat zur Gründung des Erdélyi Magyar Nemzeti Tanács/Siebenbürgischer Ungarischer Nationalrat (EMNT). Im Folgemonat wurde ein fünfköpfiges Führungsgremium besetzt, dessen Vorsitz Tibor Toró T. übernahm. Das Szeklerland erhielt mit dem Székely Nemzeti Tanács/Nationalrat der Szekler (SZNT) eine eigenständige, dem EMNT aber verbundene Struktur. Auf der Gründungsversammlung des SZNT im Oktober 2003 wählten die Delegierten József Csapó an dessen Spitze. Im Dezember 2003 schließlich wurde auch der EMNT formal gegründet. László Tőkés erhielt zugleich Präsidentenamt und Ehrenvorsitz. Die Geschäftsführung des EMNT erfolgt durch ein 18-köpfiges ständiges Komitee, an dessen Spitze Toró T. steht. Der Wunsch Béla Markós, den EMNT als neue Plattform in den Ungarnverband zu integrieren, wurde umgehend abschlägig beschieden. ${ }^{34}$

Da der EMNT in seinem Selbstverständnis keine Partei ist, beabsichtigt er nicht, bei Wahlen gegen den RMDSZ anzutreten. Die ihm nahe stehende, mit dem Ungarnverband politisch konkurrierende Partei, der Magyar Polgári Szövetség/Ungarischer Bürgerverband (MPSZ), wurde erst Ende Januar 2004 gegründet. Lediglich in Odorheiu Secuiesc/Székelyudvarhely

29 RMDSZ, PDSR: Egyezmény a Romániai Magyar Demokrata Szövetség és a Romániai Társadalmi Demokrácia Pártja között [Übereinkommen zwischen dem Demokratischen Ungarischen Verband Rumäniens und der Partei der Sozialen Demokratie Rumäniens], in: Népújság, 30.12.2000.

30 Tibor Bogdán: Belső megújulást sürget az RMDSZ reformtömörülése [Die Reformplattform des RMDSZ drängt nach innerer Erneuerung], in: Magyar Hírlap, 28.5.2001.

31 So prophezeite - unter anderen - auch Géza Szőcs, dass der RMDSZ das Jahr 2010 in seiner derzeitigen Form nicht erreichen werde (Géza Szőcs, Mária Gál (Anm. 14)); der Philosoph und Politiker Dan Oprescu vermutete im Jahre 1999, dass es dem RMDSZ ab etwa 2004/05 nicht mehr gelingen werde, seine Wählerschaft ethnisch zu organisieren (Dan Oprescu: A nemzeti kisebbségek 1999-ben [Die nationalen Minderheiten im Jahre 1999], in: Magyar Kisebbség 2/2000).

32 O.N.: »Az RMDSZ nem politikai párt...« [Der RMDSZ ist keine politische Partei...], in: TransIndex, 23.5.2001.

33 Zoltán Tibori Szabó: Az RMDSZ centrista szövetség [Der RMDSZ ist eine zentristische Vereinigung], in: Népszabadság, 5.7.2004.

34 Interview mit einem Repräsentanten des RMDSZ. Bukarest, 19.7.2001. 
hatte der MPSZ bereits seit Juli 2003 auf kommunaler Ebene bestanden. ${ }^{35}$ Auf der Gründungsversammlung erklärte der MPSZ-Vorsitzende Jenô Szász, bei den Kommunalwahlen im Juni 2004 Kandidaten gegen den RMDSZ aufstellen zu wollen - allerdings nur in solchen Kommunen, wo den Ungarn daraus keine Nachteile erwüchsen. ${ }^{36}$

\section{Fragmentierung und Einheit der Magyaren}

Die neue Konstellation zeichnete sich dadurch aus, dass mit Gründung von EMNT/SZNT und MPSZ einerseits politische Gegenspieler zum Ungarnverband entstanden, diese andererseits aber für dieselben Ziele eintraten. Die Abgrenzung des MPSZ zum RMDSZ erfolgte daher auch nur zögerlich. Da der RMDSZ dem Wunsch nicht entsprach, die Parlamentsmandate des Ungarnverbands auf beide Organisationen aufzuteilen, saßen MPSZ- bzw. EMNT-Aktivisten für den RMDSZ im rumänischen Parlament. ${ }^{37}$ Häufig traten Mitglieder der neuen Organisationen nicht einmal aus dem Ungarnverband aus oder, wie im Falle des Vizepräsidenten des EMNT, Zsolt Szilágyis, verbanden dies mit dem Wunsch, trotz des Austritts weiterhin in der Parlamentsfraktion des RMDSZ zu verbleiben. ${ }^{38}$ Zur ersten Kraftprobe im Wettstreit mit dem Ungarnverband konnte der MPSZ nicht antreten, da er aufgrund formaler Unzulänglichkeiten von der zentralen Wahlbehörde in Bukarest keine Zulassung zu den Parlamentswahlen 2004 erhielt. ${ }^{39}$ Einige seiner Aktivisten traten daraufhin als unabhängige Vertreter an.

Programmatisch forcierten die neu gegründeten Organisationen die Forderungen nach territorialen und administrativen Autonomierechten. Bereits im Juni 2003 reichten fünf dem EMNT nahe stehende RMDSZ-Parlamentarier eine Initiative im Bukarester Abgeordnetenhaus ein, die rumänische Verfassung um Rechtsansprüche auf kulturelle und regionale Autonomie zu ergänzen. Die Fraktion des RMDSZ distanzierte sich davon umgehend. ${ }^{40}$ Zur Umsetzung der Autonomiekonzeptionen erarbeitete SZNT-Präsident József Csapó, der seinerzeit auch das Autonomiekonzept für den RMDSZ entwickelt hatte, einen ausführlichen Entwurf für das Szeklerland. ${ }^{41}$ Der EMNT beauftragte eine Expertengruppe unter Leitung des Politologen Miklós Bakk, parallel eine eigene Vorlage für ein Gesetzespaket zu entwerfen. Der Ungarnverband hingegen lehnte beide als verfassungswidrig ab, sah sich aber genötigt, eine eigene Vorlage anzukündigen. ${ }^{42}$ Diese stellte er bis zum

35 Erdélyi Magyar Nemzeti Tanács: Kronológia [Chronologie]. http://www.emnt.org/ kronologia.htm

36 Zoltán Tibori Szabó: Megalakult az RMDSZ ellenszervezete [Gegenorganisation zum RMDSZ gegründet], in: Népszabadság, 4.7.2003; Gábor Miklós: Tőkés püspök busza [Der Bus des Bischofs Tőkés], in: Népszabadság, 3.2.2004.

37 O.N.: Magyar Polgári Szövetség Erdélyben [Ungarischer Bürgerverband in Siebenbürgen], in: Fidesz Hírlevél 4/2004.

38 O.N.: A párbeszéd nélkülözhetetlen, a magyar-magyar viszony vonatkozásában is [Der Dialog ist unentbehrlich, auch im ungarisch-ungarischen Beziehungsverhältnis], in: RMDSZ Tájékoztató 2765/2004.

39 O.N. Kilépett az RMDSZ-ből Szilágyi Zsolt [Zsolt Szilágyi trat aus RMDSZ aus], in: Romániai Magyar Szó, 5.6.2004.

40 O.N.: Nem érvényesek az MPSZ-t támogató aláíások? [Sind die den MPSZ unterstützenden Unterschriften ungültig?], in: Romániai Magyar Szó, 26.4.2004.

41 Erdélyi Magyar Nemzeti Tanács (Anm. 35).

42 József Csapó: Székelyföld autonómia statutum (Terra Siculorum - Ţinutul Secuiesc). Tervezet. Sepsiszentgyörgy, 2004. január 17. [Autonomiestatut des Szeklerlandes. Entwurf. Sepsiszentgyörgy, 17. Januar 2004], in: Magyar Kisebbség 1-2/2004. S. 261-294.
31. März 2005 fertig. ${ }^{43}$ Hatte sich die moderate Führung bislang darauf verständigt, Autonomien nicht mehr ostentativ öffentlich zu fordern, sondern eine Politik der kleinen Schritte zu verfolgen, enthielt der Entwurf nun Vorschläge für drei Autonomieformen (administrativ, kulturell, regional) und schloss lediglich territoriale Autonomien aus. Von der prinzipiellen Forderung nach territorialer Autonomie habe sich, so Árpád Márton, der für die Vorlage mitverantwortlich zeichnete, der RMDSZ aber nicht verabschiedet. ${ }^{44}$

Der Entwurf des Ungarnverbands war heftiger Kritik ausgesetzt. ${ }^{45}$ Dies deutet auf das Dilemma des RMDSZ hin: Erstmals warb eine oppositionelle Kraft um dieselbe Klientel. Beide gingen von einer kommunitaristischen Grundkonzeption aus und verstanden die ungarische Gemeinschaft als Kollektivsubjekt. Die diese Konzeption nicht teilenden individualistischen Strömungen 3 und 4 waren auch im Ungarnverband in der Minderheit. Eine von einem Vertreter dieser Gruppe, Sándor Szilágyi N., in den 1990er Jahren entwickelte Vorlage für ein Minderheitengesetz, der den Minderheiten keine Sonderrechte zuweisen wollte, sondern nach einer für alle Staatsbürger geltenden Regelung strebte, verschwand seinerzeit sofort wieder von der Tagesordnung. ${ }^{46}$ Die kollektivistischen Positionen wurden nach den Abspaltungen durch weit reichende Forderungen, bekannte Führungspersönlichkeiten und populistische Rhetorik der Radikalen zusätzlich gestärkt. EMNT-Vertreter forderten die »vollständige Zweisprachigkeit « der Region und eine gesonderte Wahlordnung. ${ }^{47}$ László Tőkés erklärte demonstrativ: Da Grenzänderungen nicht möglich seien, müsse der Weg territorialer Autonomie beschritten werden. ${ }^{48}$

\section{Konsequenzen der Spaltung}

Infolge der Parlamentswahlen Ende 2004 trat der RMDSZ wieder in die Regierungskoalition ein und erreichte die Aufnahme seiner Forderungen nach Autonomie bzw. einem Minderheitengesetz in das Koalitionsprogramm. Bei der Umsetzung traten allerdings erneut Konflikte mit der Opposition und den Koalitionspartnern auf. Als die Regierungsvorlage im Sommer 2005 im Senat zur Debatte stand, verschleppten die Parteien zunächst eine Abstimmung und legten den Entwurf der Venedig Kommission des Europarats vor. Nachdem diese einige Modifikationen anregte, jedoch keine prinzipiellen Einwände erhob, stimmten konservative Vertreter der Koalition zusammen mit Sozialdemokraten und Nationalisten am letzten Tag, bevor die Vorlage den Senat automatisch als gebilligt verlassen hätte, gegen den Entwurf. Dies führte erneut zu einer

43 Tibor Tamás: Az RMDSZ saját autonómiatervet készít [Der RMDSZ fertigt eigenen Autonomieplan an], in: Népszabadság Online, 8.3.2004.

44 Törvénytervezet a Romániai Nemzeti Kisebbségek Jogállásáról [Gesetzesvorlage zur Rechtslage der rumänischen nationalen Minderheiten], in: Szabadság, 8.6.2005, S. 7-9.

45 O.N.: Újabb autonómia-tervezet [Neuerlicher Autonomieplan], in: Hargita Népe, 15.2.2005.

46 Vgl. Előd Kincses: Félrevezetés [Irreführung], in: Élet és Irodalom, 35/2005; Miklós Bakk, Jenó Kiss, Zoltán Gazda: Nemzet-nemzetiség: Lesz-e ebből autonómia? [Nation-Nationalität: Entsteht daraus Autonomie?]. http://www.mtaki.hu/docs/romaniai_kisebbsegi_torvenytervezet/ lesz_e_ebbol_autonomia_050412_m.htm

47 Vgl. Sándor Szilágyi N., Kriszta Székely: Kényszerpályán az RMDSZ? [Ist der RMDSZ auf einem Zwangspfad?], in: Szabadság 26.3.2005, S. 5.

48 Erdélyi Magyar Nemzeti Tanács (Anm. 35). 
Regierungskrise. ${ }^{49}$ Béla Markó erklärte, wenn keine Einigung erzielt werden könne, bliebe nur die Möglichkeit von Neuwahlen.

Auch nachdem der liberale Premier Tariceanu das Gesetz im November erneut auf den Weg gebracht und der RMDSZ einige der von der Venedig Kommission bemängelten Passagen modifiziert hatte, währte der Widerstand in der Koalition fort. Die konservativen Regierungsmitglieder verlangten die vollständige Eliminierung einiger »giftiger Absätze«, insbesondere des kompletten fünften Abschnitts über kulturelle Autonomie. Die Demokratische Partei erklärte, aufgrund der Signalwirkung, die das Gesetz besitze, dürften sich darin lediglich die »europäischen Standards « widerspiegeln und nicht »Rechte, die nicht existierten «. ${ }^{50}$ Ende Januar 2006 bezog Präsident Băsescu erstmals Stellung. Vor dem Europarat betonte er, europaweit gebe es in keinem Land ein Gesetz über kulturelle Autonomie. ${ }^{51}$ Der RMDSZ-Abgeordente Kelemen Hunor kündigte daraufhin an, wenn sich die PD nicht bewege, werde der Ungarnverband deren Gesetzesinitiativen nicht mehr unterstützen.

Die Fronten zwischen den Parteien blieben offen, auch nachdem der Präsident des Europäischen Parlaments, Josep Borrell, im Februar 2006 das Minderheitengesetz ausdrücklich befürwortete und explizierte, es sei an den nationalen Regierungen, ein solches Gesetz zu verabschieden. ${ }^{52}$ Bereits im Dezember hatte das Europaparlament Formen kultureller Autonomie für Rumänien unterstützt. ${ }^{53}$ Dennoch kündigte die PD im März einen Bericht über internationale Minderheitennormen an und erklärte bereits vorab, dieser werde demonstrieren, dass die Vorlage des RMDSZ verfassungswidrig sei und gegen europäische Normen verstoße. ${ }^{54}$

\section{Interethnische Konfliktperspektiven}

Der RMDSZ war als Dachorganisation aller ethnischen Magyaren in Rumänien gegründet worden und erhebt diesen Anspruch bis heute. Regelungen bei der Einführung des ungarischen Statusgesetzes, bei dem das »Ungarische« über die Zugehörigkeit zu entsprechenden Organisationen identifiziert wurde, oder Passagen aus dem jüngsten Entwurf eines Minderheitengesetzes, das in bestimmten Fällen Konsultationspflicht »der gesetzlichen Vertreter der betreffenden Minderheiten $\ll^{55}$ verlangt, unterstützten diesen Anspruch. Verbandspräsident Markó appelliert weiterhin, der RMDSZ dürfe nicht in »links«

49 Emese Rostás-Péter: Megalakult az Erdélyi Magyar Nemzeti Tanács [Der Siebenbürgische Ungarische Nationalrat wurde gegründet], in: Romániai Magyar Szó, 15.12.2003.

50 Valentina Deleanu, Roxana Andronic: Coalition is about to break, in: Ziua, 25.10.2005.

51 O.N.: Nationalist Social Democrats, in: Ziua, 14.11.2005; Valentina Deleanu: Special commission for amendments to national minorities' status, in: Ziua, 8.12.2005.

52 Răzvan Gheorghe: UDMR threatens PD, in: Ziua, 27.1.2006.

53 O.N.: Minorities' status is in keeping with European norms and the Constitution, in: Ziua, 17.2.2006.

54 European Parliament: Resolution on the extent of Romania's readiness for accession to the European Union, P6_TA-PROV(2005)0531, adopted on 15 December 2005

55 Răzvan Gheorghe: PD elaborated national minorities' status again, in: Ziua, 21.3.2006. und »rechts« auseinander dividiert werden. ${ }^{56}$ Im Kern vertreten sowohl der RMDSZ wie auch EMNT/SZNT und MPSZ fast identische Zielvorstellungen. Beide Akteursgruppen stammen aus dem kommunitaristischen Lager. Die neuen Konkurrenten betonen daher, EMNT und MPSZ seien nicht gegen den RMDSZ gegründet worden, sondern für die Durchsetzung der Autonomierechte. ${ }^{57}$ Tökés definiert den Dialog mit dem Ungarnverband als eines der beiden wichtigsten Ziele und ist überzeugt, im Kern die gleichen Positionen zu vertreten. ${ }^{58}$ Ebenso stellt der Parlamentarier Ferenc Pécsi heraus, man wolle nicht Konkurrenz, sondern Unterstützung für den RMDSZ. ${ }^{59}$ Dennoch erschwert die Existenz der neuen Gruppierungen die Politik des Ungarnverbands. Bereits vor der Abspaltung übten die radikaleren Kräfte im Verband einen beträchtlichen Druck auf die Moderaten aus und beschränkten deren Kompromissfähigkeit. Die Abspaltung ohne echte Konkurrenz verschärft den Druck auf den RMDSZ, programmatische Zielsetzungen nicht zu vernachlässigen. Die magyarische Bevölkerung Siebenbürgens teilt die moderate Linie des Verbands keineswegs uneingeschränkt. ${ }^{60}$ Die Vorlage zweier Autonomiekonzeptionen durch SZNT und EMNT zwang den RMDSZ daher, die seit 1995 vernachlässigten programmatischen Debatten ${ }^{61}$ wieder aufzunehmen und sich mit einem eigenen Entwurf zu positionieren.

Im Parteienspektrum Rumäniens nimmt der Ungarnverband eine Sonderstellung ein. Seine Forderungen nach Dezentralisierung und Autonomie führten in regelmäßigen Abständen zu eklatanten Konflikten. Die rumänischen Parteien weigern sich, die Multinationalität Rumäniens anzuerkennen, wodurch die Handlungsfähigkeit der Regierungen, an denen der Ungarnverband seit zehn Jahren kontinuierlich entweder direkt oder indirekt beteiligt ist, erheblich beeinträchtigt wird. Mit den neuen Organisationen wuchs der Druck auf den RMDSZ, und er bräuchte Partner, um mit seiner moderaten Politik die ethnischen Interessen seiner Wählerschaft befriedigen zu können. Die Grenzen einer solchen Politik sind aber klar gesteckt. Vor allem die größten Parteien der beiden Lager, die Sozialdemokratische und die Demokratische Partei, zeigen sich kompromisslos in ihren Positionen. Der frühere Ministerpräsident Năstase ist erklärter Gegner von ethnisch definierten Rechten, die die Einheit von Staat und Nation gefährden könnten. ${ }^{62}$ Eine offizielle Verlautbarung der PSD sprach nach der Gründung des EMNT von »ungarischem Irredentismus « sowie »aggressiven und gefährlichen Aktionen«,

56 Törvénytervezet a Romániai Nemzeti Kisebbségek Jogállásáról (Anm. 42), II, 1 (18).

57 A RMDSZ célja erôs parlamenti képviseltet létrehozni az ôszi választások során - Szövetségi Képviselõk Tanácsa - Marosvásárhely, 2004. július 3 [Das Ziel des RMDSZ ist eine starke parlamentarische Vertretung im Zuge der Wahlen im Herbst - Rat der Verbandsrepräsentanten - Marosvásárhely, 3. Juli 2004], in: RMDSZ Tájékoztató, 2763/2004.

58 So der Vizevorsitzende Sziláygi (Emese Rostás-Péter (Anm. 49)).

59 O.N.: Elvi nyitottság a párbeszédre. Tőkés László megbeszélést kezdeményez az RMDSZ vezetőivel [Prinzipielle Dialogbereitschaft. László Tőkés initiiert Gespräch mit RMDSZ-Führung], in: Szabadság, 30.12 2003. S. 1; László Tőkés, Cristian Unteanu: Tőkes: Basescu legitimated Magyars' automony, in: Ziua, 24.3.2006.

60 Lajos Sike: Kifütyölték a másféle véleményt!? [Pfiffen sie die andersartige Meinung aus!?], in: Romániai Magyar Szó, 6.12.2003.

61 Hintergrundgespräch mit einem Vertreter der ungarischen Rumänen. Krefeld, 27.11.2004.

62 Vgl. Adrian Năstase: Sovereign Nation-State between Integration and Autonomy, in: Romanian Journal of International Affairs 3-4/1998. S. 19-27. 
und der Vorsitzende der PD, Emil Boc, warnte vor "gefährlichen Präzedenzfällen «. ${ }^{63}$

In der Krise um György Frunda spiegelt sich das Dilemma des rumänischen Ungarnverbands, der Erfolge verbuchen muss, um gegen seine politischen Konkurrenten zu punkten. Die

63 O.N.: Az SZDP szerint az EMNT a »Magyar Irredentizmus Tanácsa « [Die PSD betrachtet den EMNT als »Rat des ungarischen Irredentismus «], in: Romániai Magyar Szó, 18.12.2003.
Fundamentalopposition, die ihm in der Regierungskoalition aus den Reihen der PD entgegenschlägt, lässt derzeit eher Neuwahlen als einen Kompromiss wahrscheinlich erscheinen. Sollten sich die radikalen ungarischen Vertreter bei einer Parlamentswahl gegen den RMDSZ etablieren können oder dazu beitragen, dass keine der ungarischen Organisationen mehr im Parlament vertreten sein wird, dürfte dies die Konfliktlage in Rumänien verschärfen.

FOR U M

\title{
In the Name of the Pater, or Why Democracy Remains Absent from Central Asia
}

\author{
Klavdija Černilogar*
}

\begin{abstract}
The independence of the Central Asian countries has not been accompanied by transition towards democracy. This process is prevented through the dominance of neopatrimonial patterns of governance, which combine new formally existing democratic institutions and the traditional persistence of the patrimonial system of rule. Two basic characteristics of this type of rule are its rootedness in the traditional societal structure and the misuse of the imprecise legal framework. The international community's attempts to contribute to the democratisation of Central Asia are largely unsuccessful and will remain so until there is clear domestic preparedness for reform.
\end{abstract}

Keywords: Central Asia, neopatrimonialism, democracy

Contrary to nationalist movements that resulted in the establishment of independent states in Central and Eastern Europe in the early 1990s, the independence of Central Asian states was not strived for from within; instead, it was the consequence of the Soviet Union's dissolution. After the change, it was no longer enough for the incumbents to please Russia to stay in power; now they had to secure their political existence on their own. ${ }^{1}$ Kazakhstan's and Uzbekistan's presidents have extended their mandates through nation-wide referenda. In short, they have managed to manipulate the existing procedural options to serve their personal interests. In the 15 years of independence of the five Central Asian countries, practically only five presidents have ruled there. Three state presidents - the Kazakh Nursultan Nazarbayev, the Uzbek Islam Karimov, and the Turkmen Saparmurat Niyazov - have been heads of their republics since independence in 1991, coming from top positions they had occupied while still in the Soviet

\footnotetext{
* Klavdija Černilogar holds the European Master's Degree in Human Rights and Democratisation, and has done her research for the Master thesis at the Institute for Peace Research and Security Policy at the University of Hamburg (IFSH). Currently, she works as intern at the Council of Europe office in Belgrade

1 Anatoly M. Khazanov, After the USSR. Ethnicity, Nationalism and Politics in the Commonwealth of Independent States, Madison, The University of Wisconsin Press, 1995, p. 141
}

Union. Tajikistan's head Emomali Rahmonov has been in office since 1992, although formally elected as president only in 1994, and the president of Kyrgyzstan Askar Akayev ruled between 1991 and $2005 .^{2}$

Having become part of the international community as sovereign states, the five Central Asian countries formally accepted democratic standards, including those of free and fair elections, at least as members of the United Nations and the Organization for Security and Co-operation in Europe. The latter has conducted several election observation missions in all but Turkmenistan, and has repeatedly concluded that the elections were not in accordance with its commitments. Other outside assistance on the way to democratisation, offered by countries as well as international governmental and non-governmental organisations, also seems to be in vain. Why are external efforts fruitless? Why does the rule of the pater persist? We will attempt to answer these two questions below; the former through a theoretical approach to democratisation, the latter through an analysis of the Central Asian political environment. 2 For details on the political background of individual presidents, see Martha
B. Olcott, Taking Stock of Central Asia, in "Journal of International Affairs«, vol. 56, no. 2, 2003, pp. 8-9. 